\title{
Maximum Likelihood Estimation of Diffusion and Convection in Tokamaks using Infinite Domains
}

\author{
Matthijs van Berkel ${ }^{1,2}$, Gerd Vandersteen ${ }^{3}$, Hans Zwart ${ }^{1}$, Dick Hogeweij ${ }^{2}$, Marco de Baar ${ }^{1,2}$
}

\begin{abstract}
A new method to identify the spatial dependent parameters describing the heat transport, i.e. diffusion and convection, in fusion reactors is presented. These parameters determine the performance of fusion reactors. The method is based on local transfer functions, which are defined between two measurement locations. Estimation of the local transfer functions results in a model of the spatial dependent diffusion and convection. The parameters of the local transfer functions are estimated using Maximum Likelihood Estimation in the frequency domain. This is necessary, because both measurements (input and output of the transfer function) contain noise. Moreover, confidence bounds and validation tests can be used in this framework. Finally, experimental results are presented, which show that the diffusion and convection can be estimated. In this case, the uncertainty bounds are significant on the convection.
\end{abstract}

\section{INTRODUCTION}

In the future, sustainable, clean, and safe power plants are needed. A possible future energy source that fulfills these requirements is nuclear fusion, in which hydrogen isotopes are fused to produce abundant amounts of energy. The most successful nuclear fusion reactor to date is the tokamak.

A tokamak uses magnetic fields to confine a plasma (ionized gas). The magnetic field-lines to which the one-dimensional transport is perpendicular form a topology of flux-surfaces, which the charged particles are forced to follow [1]. Therefore, it is difficult for particles to move from one flux surface to another. Consequently, the transport of particles and heat from the center to the wall is limited. Unfortunately, this magnetic confinement is not perfect

\footnotetext{
${ }^{1}$ Eindhoven University of Technology, Dept. of Mechanical Engineering, Control Systems Technology group, PO Box 513, 5600 MB Eindhoven, The Netherlands (e-mail: m.v.berkel@tue.nl)

${ }^{2}$ FOM institute DIFFER, Dutch Institute for Fundamental Energy Research, Association EURATOM- FOM, Trilateral Euregio Cluster, PO Box 1207, 3430 BE Nieuwegein, The Netherlands

${ }^{3}$ Vrije Universiteit Brussel, Department of Fundamental Electricity and Instrumentation, Pleinlaan 2, 1050 Brussels, Belgium
}

due to turbulence inside the plasma, thus limiting the performance of fusion machines [2], [3].

Experimental evidence suggests that this turbulent transport is associated with the distribution of the angular momentum and the current density inside the plasma [4]. These distributions can be manipulated (locally) using a number of actuators such as neutral beam injection, electron current drive [5], [6]. It is shown that by manipulating these distributions, the performance of tokamaks can be significantly increased [7]. However, to study the effect of these actuators on the thermal transport requires modeling and estimation of this transport.

Thermal transport can be described as the effective thermal diffusivity, $D(\rho)$ and the convective velocity, $V(\rho)$ [8], [9]. These quantities are functions of the plasma minor radius which is expressed in the normalized radius $\rho$ [2]. A well established method to measure the thermal transport is the analysis of heat pulses in the plasma induced by localized deposition of modulated power. Their propagation and their dispersion carry the information to deduce the profiles: $D(\rho)$ and $V(\rho)$ [8], [10]. These methods, however, do not take the effect of noise into account and usually neglect convection.

In this contribution, we will develop an alternative method to estimate the profiles of $D(\rho)$ and $V(\rho)$. The merit of this approach is that, contrary to [8], [10] the new method includes the convection and takes the noise into account. Non-rational SingleInput Single-Output (SISO) transfer functions can be defined between two measurement locations due to the infinite domain description. Consequently, only SISO transfer functions that depend on two parameters per location need to be estimated. Another advantage of this local modeling is that disturbances or non-linearities at other locations are less likely to influence the estimation. Here, only local transfer functions are estimated on locations where no source term is present due to the uncertainty of the source 
term itself.

The noise is taken into account by using Maximum Likelihood Estimation in the frequency domain allowing the use of non-rational transfer functions. As a consequence, the method allows for a systematic estimate of the spatial dependent diffusion and convection including confidence bounds. More importantly, the resulting models can also be validated or rejected using a number of tests.

This article is structured as follows: Section 2, describes the heat transport and modeling inside tokamaks. Also the sensors and the actuator used are explained. Then, the local transfer functions used for the identification of the parameters are derived. In Section 3, the Maximum Likelihood Estimation scheme is explained. In addition, the optimization and validation methodologies are discussed. Section 4 shows experimental results including the validation tests introduced in Section 3. Finally, a number of conclusions are summarized and discussed.

\section{THERMAL TRANSPORT INSIDE TOKAMAKS}

In this section, the experimental set-up is described such as the sensors and microwave source. Then, it is explained how thermal transport in tokamaks can be modeled.

\section{A. Excitation and Sensors}

A plasma can be locally heated by means of Electron Cyclotron Resonance Heating (ECRH) (microwave heating) [2]. The resonance frequency and the angle of the mechanical steering mirror are used to position the heat source on the minor radius. The local ECRH power-density can be modulated to create heat pulses which perturb the plasma and allow for the identification of the diffusion and convection. However, most gyrotrons are limited to block wave modulation.

The resulting temperature fluctuations are measured by means of Electron Cyclotron Emission (ECE). Measuring the fluctuation of intensity in a small interval of the gyration frequency gives the temperature perturbation on that location [11]. The gyration frequency depends on the magnetic field $B$, which depends on the radius via $B \sim 1 / R$. Therefore, it is possible to measure the temperature at many locations in the plasma.
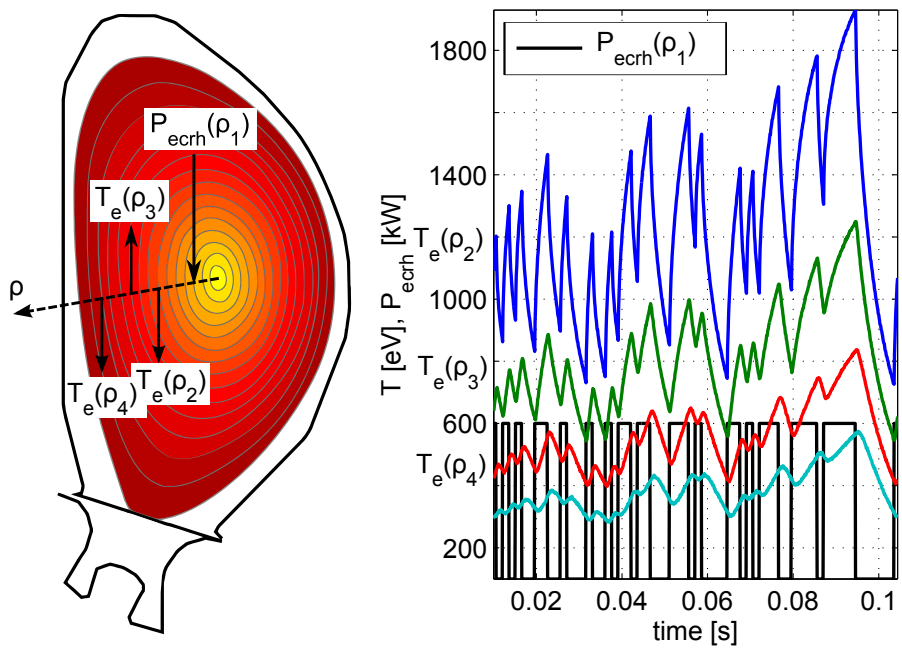

Figure 1. Graphical representation of nested flux surfaces inside a Tokamak, heat source and measurement channels (left). Simulation of temperature fluctuations at different measurement locations due to the heat-source (right).

A graphical depiction of the flux surfaces and temperature fluctuations due to a heat-source is presented in Fig. 1.

The dominant measurement noise contribution on ECE is the so-called thermal noise, which originates from the fact that not all electrons gyrate with the same frequency but rather have a normal distribution at the considered flux surface [12]. This also means that the thermal noise is additive normally distributed noise and therefore will be modeled as such.

\section{B. Mathematical description}

Inside a tokamak different transport mechanisms determine the heat transport. These consist of electrical, particle, and the thermal transport of both ions and electrons. The electron heat transport is the fastest transport and is therefore considered as the important transport. Moreover, the other quantities like density and electric fields can be considered in steady-state $\left(\frac{\partial}{\partial t}=0\right)$ when only the electron temperature $\left(T_{e}\right)$ is perturbed by ECRH.

A tokamak has a special magnetic topology of nested flux surfaces (the ellipsoids in Fig. 1). This allows the heat-transport to be modeled as a onedimensional heat equation in cylindrical coordinates [9]. In spite of specific toroidal effects, the plasma can be modeled to sufficient accuracy as a cylinder 
by introducing a dimensionless minor radius $\rho$

$$
\begin{aligned}
\frac{\partial T_{e}}{\partial t}=\frac{1}{\rho} \frac{\partial}{\partial \rho} & \left(r D(\rho) \frac{\partial T_{e}}{\partial \rho}+r V(\rho) T_{e}\right) \\
& +P_{\text {ecrh }}(\rho, t) .
\end{aligned}
$$

At the plasma center $\rho=0$ and at the last closed flux surface $\rho=1$ (see Fig. 1). Therefore, knowledge of the magnetic topology is necessary to transform the absolute radius into the normalized radius. The ECRH power is denoted by $P_{\text {ecrh }}$; it is locally applied and modulated in time. In the literature, [8], [10], (1) is simplified when the heat transport is analyzed in the intermediate region between center and the wall of the tokamak. The reason is that in this region the radial effects are less strong such that transport can be approximated by a linear heat equation with varying coefficients

$$
\frac{\partial T_{e}}{\partial t}=D(\rho) \frac{\partial^{2} T_{e}}{\partial \rho^{2}}+V(\rho) \frac{\partial T_{e}}{\partial \rho}+P_{e c r h}(\rho, t) .
$$

This model forms the basis for our estimation of the diffusion $D(\rho)$ and convection $V(\rho)$. It is important to note that the heat equation (2) describes many other processes in physics as well, such as heat transfer in solids and fluids [13], [14]. In the next section transfer functions are derived allowing the modeling of the diffusion and convection.

\section{LOCAL MODEL DESCRIPTION}

The spatial dependent diffusion and convection in equation (2) need to be estimated. Here, an approach is chosen whereby the coefficients are estimated by defining a transfer function between two measurement locations. By defining such a transfer function between all consecutive measurement locations and estimating the parameters for every transfer function allows for the reconstruction of $D(\rho)$ and $V(\rho)$. This modeling has some similarity to methods used in fusion [8], [10], however, this type of modeling also includes the estimation of convection. In this section the derivation of the transfer function is explained.

\section{A. General solution in the Laplace domain}

The heat-equation (2) only needs to describe local domains. Therefore, it is assumed that the parameters are constant between two measurement locations which are close to each other. Moreover, the heatsource $P_{\text {ecrh }}$ is localized such that almost all domains do not contain this source term. At the domain(s) containing the source domain it is difficult to estimate the parameters as the exact deposition of the heat source is unknown. Hence, only sourceless domains will be considered. In addition, the Laplace transform is applied to (2) resulting in the complex valued Ordinary Differential Equation (ODE)

$$
s \Theta(\rho, s)=D \frac{d^{2}}{d \rho^{2}} \Theta(\rho, s)+V \frac{d}{d \rho} \Theta(\rho, s),
$$

where $s$ is the Laplace variable and $\Theta$ the Laplace transform of $T_{e}$ [15]. The general solution of (3) is

$$
\begin{gathered}
\Theta(\rho, s)=A_{1}(s) \exp \left(\lambda_{1} \rho\right)+A_{2}(s) \exp \left(\lambda_{2} \rho\right) \\
\text { with } \quad \lambda_{1,2}=\frac{-V}{2 D} \mp \sqrt{\left(\frac{V}{2 D}\right)^{2}+\frac{s}{D}} .
\end{gathered}
$$

The (spatial) constants $A_{1}(s)$ and $A_{2}(s)$ are determined by the choice of the boundary conditions. This choice and its consequences will be explained next.

\section{B. Infinite domain description}

The boundary conditions should be chosen such that the most local description is achieved. One boundary condition is chosen to be the temperature at the spatial location of the measurement point $\rho_{i}$, i.e. one of the measurement points. The second boundary is chosen as infinity. Consequently, only two measurements will be necessary to estimate the parameters.

An infinite domain boundary condition is defined as follows, if $\rho \rightarrow \infty$, then $\Theta \rightarrow 0$. This means that at $\rho=\infty$ all perturbations need to have vanished. Since, we follow the standard convention that the $\arg (z) \in(-\pi, \pi]$ and that $\arg (\sqrt{z})=\frac{1}{2} \arg (z)$, $z \in \mathbb{C}$, we can see that for $\rho \rightarrow \infty, \exp \left(\lambda_{1} \rho\right) \rightarrow 0$ and $\exp \left(\lambda_{2} \rho\right) \rightarrow \infty$. Therefore, $A_{2}(s)$ should be equal to zero, otherwise the solution (4) would not converge to zero $\rho \rightarrow \infty$. At location $\rho_{i}$ the temperature is $\Theta(\rho)=\Theta\left(\rho_{i}\right)$ such that

$$
A_{1}(s)=\exp \left(-\lambda_{1} \rho_{i}\right) \Theta\left(\rho_{i}\right) .
$$

Then, the temperature at the consecutive measurement location $\Theta\left(\rho_{i+1}\right)$ is considered. Now, the nonrational transfer function is given by

$$
H(\theta, s)=\frac{\Theta\left(\rho_{i+1}\right)}{\Theta\left(\rho_{i}\right)}=\exp \left(\lambda_{1}\left(\rho_{i+1}-\rho_{i}\right)\right) .
$$


The parameters of the transfer function will be estimated for all measurement locations making it possible to reconstruct $D(\rho)$ and $V(\rho)$. This local transfer function description has a number of advantages: 1) It gives the most local estimate as only measurements at two spatial locations are necessary; 2) the resulting transfer function has been significantly simplified because $\left.A_{2}(s)=0 ; 3\right)$ only SISO transfer functions need to be estimated. On the other hand, it has a few disadvantages: 1) it is assumed that the parameters are constant everywhere even outside the domain $(\rho \rightarrow \infty)$. Thus, if the parameter varies outside the considered domain then a bias will be introduced; 2) when domains are close to the real boundary, a difference between the modeled and real boundary will introduce a bias. However, the introduced bias due to miss-modeling is partly suppressed in practice. This is because (5) acts as a low-pass filter. The amount of suppression depends on the distance of the domain to the boundary. It also depends on the fluctuation of the parameters outside the domain and the distance of this fluctuation to the considered domain. These errors will be more present in the low-frequency information as high-frequency errors are suppressed more strongly.

Summarizing, these errors will only be present when considering fast changing profiles and when domains are close to the boundary. However, there is a more important issue and that is the effect of noise. The temperatures will be measured including a significant amount of noise because it is measured by means of ECE (see Section II-A). In the next section is explained how to handle this noise such that a consistent estimate of (5) can be achieved.

\section{PARAMETER Estimation}

The measurements at two neighboring locations are used to estimate the local parameters. However, both measurements are prone to noise, which means that the real temperatures are unknown. If these measurements are considered as the real temperatures this would result in a bias when applying standard estimation schemes e.g. least-squares estimation [16].

Therefore, an Errors-in-Variables (EIV) approach is used since it considers the noise spectra on both measurements including possible correlations. The EIV treats the real noiseless temperatures as unknown parameters, connected by the parametric transfer function. If the connection between the parametric transfer function and the noise variances from the spectra are known, it is possible to eliminate these unknown parameters. However, to estimate the noise variances it is necessary to have information about the Probability Density Function (PDF) of the noise. Based on physical knowledge, the dominant measurement noise is additive normally distributed noise in the time domain. This results in additive circular complex normally distributed noise in the frequency domain [17].

In addition, periodic measurements (in time) are used such that the average and variance per frequency line can be determined. Thereby, estimating the deterministic spectra and the noise spectra. Note, that this is under the assumption that the number of periods is high enough.

\section{A. Maximum Likelihood Estimation}

The Maximum Likelihood Estimator (MLE) is based on the properties of the noise spectra. Therefore, a consistent estimate of the parameters of the transfer function can be achieved even in the presence of noise. The MLE cost function is derived on the basis of the PDF and the transfer function. The MLE cost function basically requires that the resulting PDF of the temperature minus the simulated temperature is white. As the MLE cost function is infeasible to minimize, the log likelihood cost function $V_{M L}$ is used [16]. It has the same global minimum and is defined as

$V_{M L}=\frac{1}{F} \sum_{k=1}^{F} \frac{\left|\hat{\Theta}\left(\rho_{i+1}, \Omega_{k}\right)-H\left(\theta, \Omega_{k}\right) \hat{\Theta}\left(\rho_{i}, \Omega_{k}\right)\right|^{2}}{\sigma_{e}^{2}\left(\theta, \Omega_{k}\right)}$,

with $\Omega_{k}$ the excited frequency lines, $\hat{\Theta}$ the average over the periods, $F$ the number of frequencies used, and $\theta=[D, V]$. The variability is given by

$$
\begin{gathered}
\sigma_{e}^{2}\left(\theta, \Omega_{k}\right)=\sigma_{i+1}^{2}+\sigma_{i}^{2}\left|H\left(\theta, \Omega_{k}\right)\right|^{2} \\
-2 \operatorname{Re}\left(\sigma_{i+1, i}^{2} \overline{H\left(\theta, \Omega_{k}\right)}\right)
\end{gathered}
$$

where $\sigma_{i+1}^{2}$ and $\sigma_{i}^{2}$ are the variances estimated over the different periods of $\Theta\left(\rho_{i+1}\right)$ and $\Theta\left(\rho_{i}\right)$ respectively. The estimated covariance between these two noise spectra is denoted as $\sigma_{i+1, i}^{2} . \bar{H}$ is the complex 
conjugate of $H$. The parameters are estimated by minimizing the log likelihood cost function $V_{M L}$

$$
\hat{\theta}=\underset{\theta}{\arg \min } V_{M L}\left(\Omega_{k}, \theta\right) .
$$

Minimizing the resulting cost function gives the estimated parameters. The cost function (6) can be naturally interpreted as a weighting of the error with the uncertainty of the measurements. Frequency lines with a small variance have a higher weighting than frequency lines with a high variance. The uncertainty on the frequency lines can be mapped to the uncertainty of the parameters via a first order Taylor expansion based on [16]

$$
\begin{gathered}
\operatorname{Cov}(\hat{\theta}) \approx \operatorname{Re}\left(2 J_{\theta}^{H} J_{\theta}\right)^{-1} \text { with } \\
J_{\theta}=\left.\frac{\partial}{\partial \theta}\left(\frac{\hat{\Theta}\left(\rho_{i+1}, \Omega_{k}\right)-H\left(\theta, \Omega_{k}\right) \hat{\Theta}\left(\rho_{i}, \Omega_{k}\right)}{\sigma_{e}\left(\theta, \Omega_{k}\right)}\right)\right|_{\theta=\hat{\theta}} .
\end{gathered}
$$

Minimizing (6) results in the parameter estimates and (9) allows the construction of the corresponding uncertainty bounds.

\section{B. Optimization}

The minimization of the cost function (6) is a nonconvex problem, because of the non-rational transfer function and the division by the parameter dependent variability $\sigma_{e}^{2}\left(\theta, \Omega_{k}\right)$. However, the transfer function has a simple form and only depends on two variables. Therefore, it is easy to minimize the cost function. Not only standard gradient methods are applicable but also more advanced algorithms, which do not require the start values to be in the neighborhood of the global minimum [18]. In addition, a parameter transformation can be used to change the gradient map resulting in a wider region of convergence of gradient based methods.

Here, we have chosen for a Levenberg-Marquardt minimization scheme, a modified Newton-Gauss gradient method, which uses the Jacobian presented in (9) and a variable step size [19].

\section{Validation}

Two tests will be used to validate the model: a cost function analysis and a whiteness residual test. In addition, the transfer function can be estimated on the basis of data and can be compared to the transfer function based on estimated parameters. In principle, a different data set should be used for the validation. However, due to the limited data the same data set will be used.

The cost function analysis is used to detect model errors. If the noise is indeed normally distributed, no model errors are present, and if a number of (weak) assumptions are fulfilled [16], the expected value of (6) at the global minimum $\hat{\theta}$ i.e. $\mathbb{E}\left\{V_{M L}(\hat{\theta})\right\}=$ $V_{\text {noise }}$ equals approximately

$$
V_{\text {noise }} \approx\left(F-\frac{n_{\theta}}{2}\right) \text {. }
$$

with $n_{\theta}$ the number of free real-valued parameters. In addition, the variance of $V_{M L}(\hat{\theta})$ should also equal $V_{\text {noise }}$ i.e. $\operatorname{var}\left\{V_{M L}(\hat{\theta})\right\} \approx V_{\text {noise }}$. Hence, confidence bounds can be constructed such that $V_{M L}(\hat{\theta})$ resides between these bounds. Model errors generally lead to a higher value of the cost function at the global minimum.

The whiteness residual test is used to test the amount of deterministic signal information left in the residual. In other words, if all dynamics has been captured by the model, then the residual should contain no correlation over the different frequency lines i.e. the residuals should be white. The correlation residuals are calculated as defined in [20]

$$
\begin{gathered}
\hat{R}_{\delta \delta}(m)=\frac{1}{F-m} \sum_{k=1}^{F-m} \delta\left(\Omega_{k}, \hat{\theta}\right) \overline{\delta\left(\Omega_{k+m}, \hat{\theta}\right)} \\
\text { with } \quad \delta\left(\Omega_{k}, \hat{\theta}\right)=\frac{\hat{H}_{M L}\left(\Omega_{k}\right)-H\left(\Omega_{k}, \hat{\theta}\right)}{\hat{\sigma}_{H}\left(\Omega_{k}\right)} .
\end{gathered}
$$

If all transients are neglected (died out), then $\hat{H}_{M L}\left(\Omega_{k}\right)=\hat{\Theta}\left(\rho_{i+1}\right) / \hat{\Theta}\left(\rho_{i}\right)$ and $\hat{\sigma}_{H}\left(\Omega_{k}\right)$ can be calculated by

$$
\begin{aligned}
\hat{\sigma}_{H}=\left|\hat{H}_{M L}\left(\Omega_{k}\right)\right|^{2}\left\{\frac{\sigma_{i+1}^{2}}{\left|\hat{\Theta}\left(\rho_{i+1}\right)\right|^{2}}+\frac{\sigma_{i}^{2}}{\left|\hat{\Theta}\left(\rho_{i}\right)\right|^{2}}\right. \\
\left.-2 \operatorname{Re}\left(\frac{\sigma_{i+1, i}^{2}}{\hat{\Theta}\left(\rho_{i+1}\right) \hat{\hat{\Theta}\left(\rho_{i}\right)}}\right)\right\}
\end{aligned}
$$

Hence, some correlation will be present in the residual. Therefore, uncertainty bounds on the correlation 


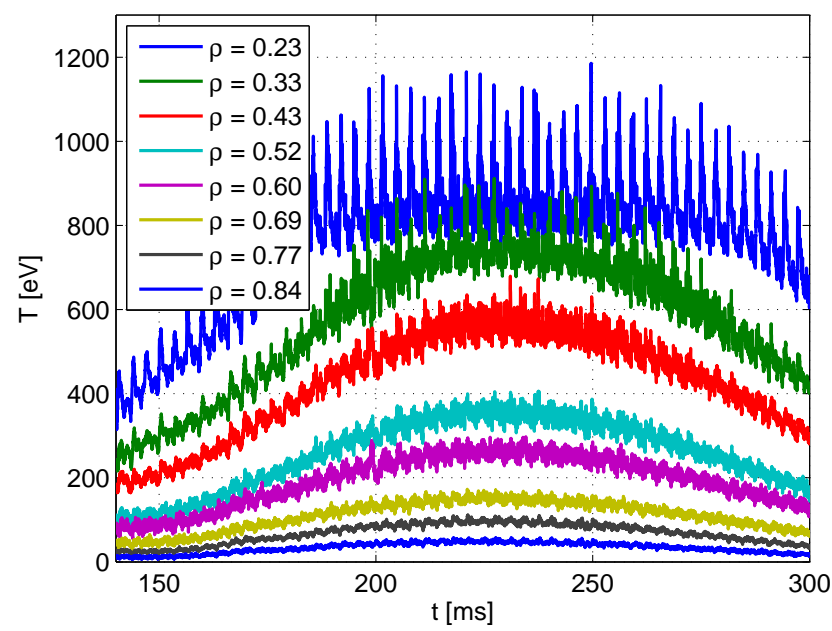

Figure 2. Temperature measurements at the different locations in RTP in terms of the normalized radius $\rho$ (Discharge r19960403.006). The amplitude of the perturbations decreases with the distance to the source. Hence, it is also clear that the ECRH is localized near the center of the Tokamak. (color online)

residuals need to be constructed once more, which is explained in [20]. Both, the value of the cost function and whiteness residual test give insight in how well the model describes the measurement data set at hand.

\section{EXPERIMENTAL RESULTS}

The MLE identification method is applied to estimate the local parameters at different locations. A real discharge (measurement) from a tokamak will be analyzed and the results will be validated and discussed.

\section{A. Set-up RTP tokamak}

The measurement data to be analyzed is acquired from the Rijnhuizen Tokamak Project (RTP). RTP is a tokamak with major radius $R_{0}=0.72 \mathrm{~m}$, which is the distance from the center of the tokamak to the plasma center. The minor radius $a=0.16 \mathrm{~m}$ is the distance from the plasma center to the wall of the vessel. The toroidal magnetic field can be up to $2.5 \mathrm{~T}$ [21]. The modulated power applied to the discharge analyzed is $P_{\text {ecrh }}=0.6 \mathrm{MW}$ with a duty cycle of $20 \%$ and a period of $3.2 \mathrm{~ms}$.

The resulting temperature fluctuations are shown in Fig. 2 in terms of normalized radii. These ECE temperature measurements are used to estimate the parameters $D$ and $V$ in terms of normalized radius $\rho$.
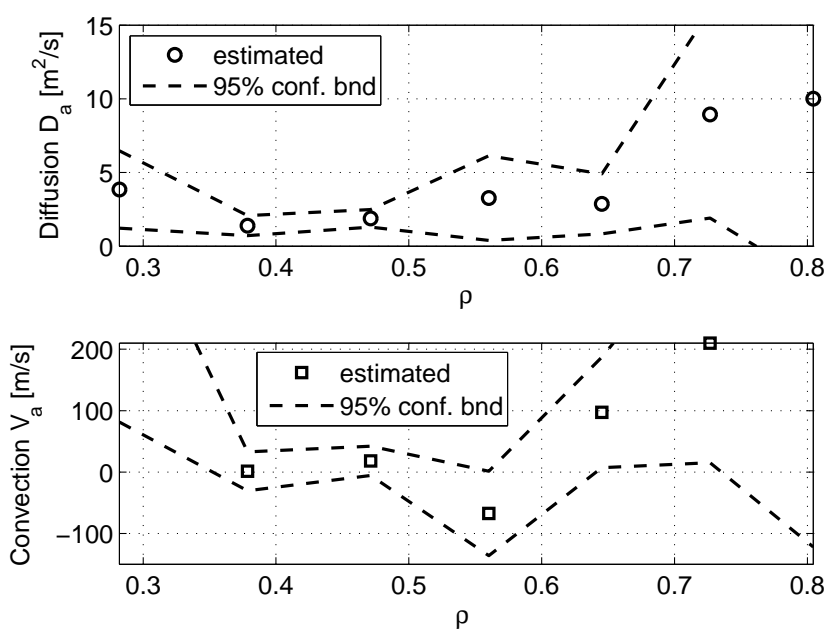

Figure 3. Estimated $D_{a}$ and $V_{a}$ with corresponding uncertainty bounds where the estimates are plotted at $\left(\rho_{i+1}+\rho_{i}\right) / 2$ (some estimates outside plotted intervals).

Note, that the central measurements are discarded as they contain the source. The full power modulation time window is included in the estimation process, including the initial heating phase and the final phase where the peripheral channels cool down due to a ramp-down of the plasma current, because this information also contributes to better estimates compared to decreasing the interval.

\section{B. Estimates of $D$ and $V$}

The estimates are calculated in terms of the normalized radius $\rho$, however, this means that the diffusion and convection are also dimensionless. Therefore, in fusion it is common practice to rescale the parameters with the minor radius such that they are expressed in SI units again i.e. $\rho=r / a$. This results in $D_{a}=a^{2} \cdot D$ and $V_{a}=a \cdot V$. However, this also requires the uncertainty bounds to be rescaled using propagation of uncertainty $\operatorname{Cov}\left(D_{a}, V_{a}\right)=$ $J_{a} \operatorname{Cov}(\hat{\theta}) J_{a}^{T}$ with $J_{a}=\left[a^{2} 0 ; 0 a\right]$. The dimensionless radius is $\rho$ retained instead of representing in terms of $r$.

Fig. 3 presents the estimates, using (8) at the excited frequency lines $\Omega_{1: 6}=$ $312.5,625,937.5,1250,1562.5 \mathrm{~Hz}$. The uncertainty of the convection estimates seem rather high compared to the signal to noise ratios (SNR) achieved on the measurements (see Table I for the SNR of the first three harmonics). Note, however, 
Table I

SNR IN DB OF THE FIRST THREE HARMONICS AT DIFFERENT $\rho$.

\begin{tabular}{|c|c|c|c|c|c|c|c|c|}
\hline$\rho$ & 0.23 & 0.33 & 0.43 & 0.52 & 0.6 & 0.69 & 0.77 & 0.84 \\
\hline \hline$\Omega_{1}$ & 27.5 & 20.7 & 22.2 & 21.6 & 21.3 & 21.8 & 20.8 & 19.4 \\
\hline$\Omega_{2}$ & 27.4 & 19.7 & 15.8 & 14.3 & 12.1 & 13.3 & 13.2 & 12.2 \\
\hline$\Omega_{3}$ & 20.2 & 11.7 & 10.3 & 6.1 & 4.9 & 7.7 & 5.8 & 7.4 \\
\hline
\end{tabular}

(a) Whiteness residual test at $\rho=0.65$

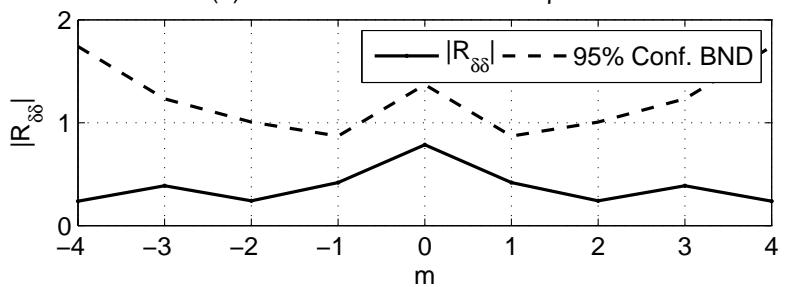

(b) Transfer function comparison at $\rho=0.65$

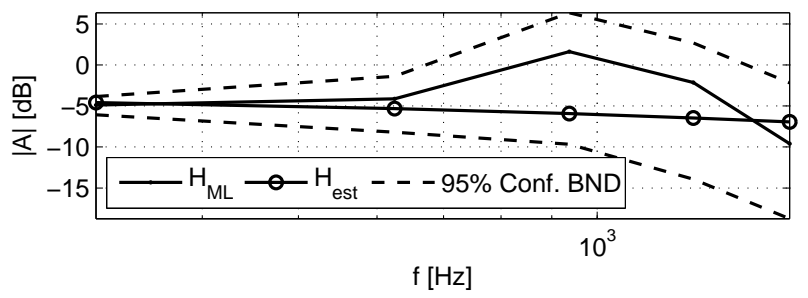

Figure 4. Whiteness residual test at $\rho=0.65$ where it is clear the model is validated. Comparison between the estimated transfer function $H_{M L}$ with its uncertainty bounds and the parametrically estimated $H$.

that both the input and output of the transfer function contain noise. Moreover, the transfer functions are non-rational, i.e. $D_{a}$ and $V_{a}$ are in the exponent, such that a small variation of amplitude can lead to a big difference in $D$ and $V$. This shows the importance of MLE estimation as it takes the noise into account when making the estimate.

\section{Validation of the estimates}

A validation procedure is necessary to determine which estimated transfer functions are a good description of the measurement data. Therefore, three tests are carried out: the cost function validation test introduced in (10); the whiteness residual test (11); and the transfer function comparison (12). The latter two are presented at one radial location in Fig. 4. The whiteness residual test validates this transfer function. In addition, the transfer function using the estimated parameters $H_{\text {est }}$ is within the uncertainty bounds of the measured transfer function $H_{M L}$. Considering, the excitation the ground harmonic has the highest amplitude and as such the lowest noise level. Therefore, the weight is stronger in the estimates (see Fig.

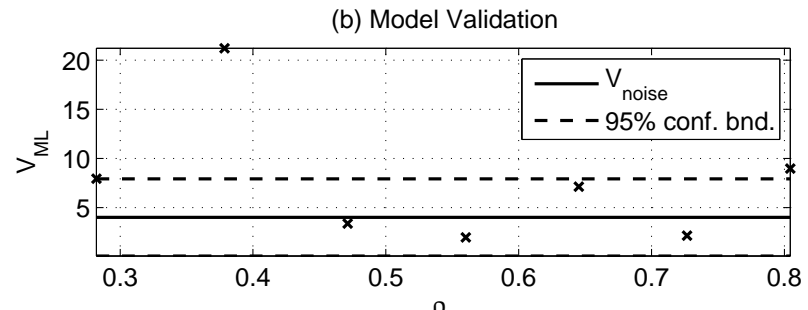

(b) Whiteness Residual Fractions

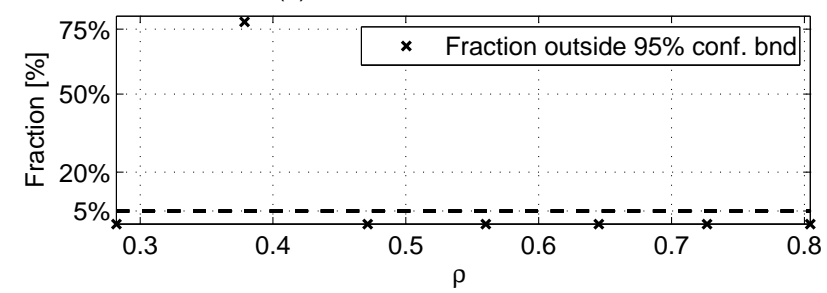

Figure 5. Validation of the estimated transfer functions using $V_{M L}$ and the fraction of $\left|R_{\delta \delta}\right|$ inside the $95 \%$ uncertainty bound. Every model which is under the uncertainty bound of $95 \%$ is validated for both the fraction plot and cost function validation. The fractions refer to the number of points outside the confidence bounds in Fig. 4(a).

3). The cost function validation test is presented and the fraction inside the $95 \%$ uncertainty bound of the whiteness residual tests for all estimates is shown in Fig. 5.

The estimates at $\rho=0.38$ and $\rho=0.81$ are rejected by the validation tests. On the other hand, the estimates at $\rho=0.28$ and between $\rho=0.48-0.73$ are validated, however, the uncertainty on the convection is high.

This is especially the case for the convection term, because convection is more sensitive to low-frequent excitation frequencies i.e. if $\omega$ is large, $D_{a}$ dominates over $V_{a}$ in (5). This is a clear indication that the chosen excitation frequency is to high. Therefore, in future experiments lower modulation frequencies could lead to better estimates. This has an additional advantage because the perturbation will have an higher amplitude and will penetrate deeper into the plasma.

\section{SUMMARY AND CONCLUSIONS}

This article presents a new methodology to estimate the spatial dependent diffusion and convection locally. This methodology is based on local transfer functions derived between two measurement locations. These transfer functions are estimated using Maximum Likelihood Estimation. This results in a consistent estimate of the parameters. The necessity 
of using a MLE estimation scheme is supported by the high uncertainty bounds on the estimated $V_{a}$. Moreover, this high uncertainty shows the importance of constructing uncertainty bounds around the estimates.

Validation tests show that not all models describe the data well and hence need to be rejected. Concluding, it is important to improve the excitation signal to arrive at better measurements by lowering the frequency range and increasing the number of excited frequencies.

\section{ACKNOWLEDGMENTS}

This work, supported by NWO, ITER- NL and the European Communities under the contract of the Association EURATOM/FOM, was carried out within the framework of the European Fusion Programme. The views and opinions expressed herein do not necessarily reflect those of the European Commission. This work is also supported by NWO-RFBR Centreof-Excellence on Fusion Physics and Technology (Grant nr. 047.018.002).

\section{REFERENCES}

[1] J. P. Freidberg, Plasma Physics and Fusion Energy. Cambridge University Press, 2007.

[2] J. Wesson, Tokamaks. OUP Oxford, 2011, vol. 149.

[3] R. Balescu, Aspects of anomalous transport in plasmas. Taylor \& Francis, 2005.

[4] F. Ryter, R. Dux, P. Mantica, and T. Tala, "Perturbative studies of transport phenomena in fusion devices," Plasma Physics and Controlled Fusion, vol. 52, p. 124043, 2010.

[5] C. Angioni, E. Fable, M. Greenwald, M. Maslov, A. G. Peeters, H. Takenaga, and H. Weisen, "Particle transport in tokamak plasmas, theory and experiment," Plasma Physics and Controlled Fusion, vol. 51, no. 12, p. 124017, 2009.

[6] J. S. deGrassie, "Tokamak rotation sources, transport and sinks," Plasma Physics and Controlled Fusion, vol. 51, no. 12, p. 124047, 2009.

[7] J. Citrin, J. F. Artaud, J. Garcia, G. M. D. Hogeweij, and F. Imbeaux, "Impact of heating and current drive mix on the iter hybrid scenario," Nuclear Fusion, vol. 50, no. 11, p. 16, Nov 2010.

[8] N. J. Lopes Cardozo, "Perturbative transport studies in fusion plasmas," Plasma physics and controlled fusion, vol. 37, p. 799, 1995.

[9] S. P. Eury, E. Harauchamps, X. Zou, and G. Giruzzi, "Exact solutions of the diffusion-convection equation in cylindrical geometry," Physics of plasmas, vol. 12, p. 102511, 2005.

[10] A. Jacchia, P. Mantica, F. De Luca, and G. Gorini, "Determination of diffusive and nondiffusive transport in modulation experiments in plasmas," Physics of Fluids B, vol. 3, no. 11, pp. 3033-3040, 1991, cited By (since 1996): 47.

[11] M. Bornatici, R. Cano, O. D. Barbieri, and F. Engelmann, "Electron cyclotron emission and absorption in fusion plasmas," Nuclear Fusion, vol. 23, no. 9, p. 1153, 1983.
[12] H. J. Hartfuss, T. Geist, and M. Hirsch, "Heterodyne methods in millimetre wave plasma diagnostics with applications to ECE, interferometry and reflectometry," Plasma Phys. Control. Fusion, vol. 39, pp. 1693-1769, 1997.

[13] H. S. Carslaw and J. C. Jaeger, Conduction of heat in solids. Clarendon Press, Oxford, 1959, vol. 1.

[14] A. Bejan and A. D. Kraus, Heat Transfer Handbook. John Wiley \& Sons, 2003.

[15] R. Curtain and H. Zwart, An Introduction to Infinite-Dimensional Linear Systems Theory. Springer-Verlag New York, 1995, vol. 21.

[16] R. Pintelon and J. Schoukens, System Identification: A Frequency Domain Approach. Wiley-IEEE Press, Hoboken (NJ), 2012.

[17] R. Gallager. (2008) Circularly-symmetric gaussian random vectors. Http://www.rle.mit.edu/rgallager/documents /CircSymGauss.pdf.

[18] S. S. Rao, Engineering optimization: theory and practice. Wiley, 2009.

[19] P. R. Gill, W. Murray, and M. H. Wright, Practical Optimization. London: Academic Press, 1981.

[20] R. Pintelon, J. Schoukens, and Y. Rolain, "Uncertainty of transfer function modelling using prior estimated noise models," Automatica, vol. 39, no. 10, pp. 1721-1733, 2003.

[21] G. Hogeweij, F. De Luca, G. Gorini, A. Jacchia, J. Konings, N. L. Cardozo, P. Mantica, M. Peters et al., "Studies of transport in the rtp tokamak," Physica Scripta, vol. 51, no. 5, p. 627, 1995. 